\title{
A Pair-Wise Key Agreement Scheme in Ad Hoc Networks
}

\author{
Woosuck Cha, Gicheol Wang, and Gihwan Cho \\ Department of Computer Science, \\ Chonbuk National University, Chonju, Korea \\ \{wscha, gcwang, ghcho\} ddcs.chonbuk.ac.kr
}

\begin{abstract}
Mobile Ad-hoc networks are exposed to various security threats because all traffics are carried in air and there is no central management authority. For the sake of secure communication in an Ad-hoc network, a scheme is inevitable to securely distribute security keys in the network. Based on the cluster structure and the verifiable secret sharing scheme[1], this paper proposes a pairwise key agreement scheme which is secure and induces a lower overhead. The proposed scheme is safe against a man-in-the-middle attack while not all private keys within the destination's cluster are exposed. In addition, our simulation result shows that the proposed scheme is more efficient and scalable than CABM(Clusterhead Authentication Based Method)[2].
\end{abstract}

\section{Introduction}

Ad-hoc networks are inherently vulnerable to security attacks because all transmissions are carried out using the opened air medium[2]. Therefore, a secure key agreement in Ad-hoc networks is more important than that of fixed networks. Since there is no TTP(Trusted Third Party), such as CA(Certification Authority) or KDC(Key Distribution Center) in Ad-hoc networks[3], all nodes are responsible of cooperative key distribution and management duty.

In this paper, a scheme is proposed to make a pair-wise key agreement between two Ad-hoc nodes securely with a lower overhead. The proposed scheme is based on the Diffie-Hellman protocol. Then each node hides its Diffie-Hellman value by encrypting with its counterpart's public key. As a result, only the counterpart can decrypt the Diffie-Hellman value. Also, the proposed scheme hides the source and destination of the key agreement so that an adversary cannot launch a man-in-the-middle attack easily while he cannot get all private keys within the destination's cluster.

\section{Related Work}

In literature [2], a key agreement scheme based on clueterhead authentication is proposed. In this scheme, two clusterheads representing their clusters respectively perform a mutual authentication using the counterpart's public key. Therefore, each clusterhead must distribute its public key to all other clusterheads. After the authenti- 
cation between two clusterheads, a pair-wise key is exchanged between them, and eventually the key is distributed to the communicating members. Because this scheme forces each clusterhead to distribute its own public key to all other clusterheads, it causes high communication overhead. Also, since a communicating node must know the cluster to which its counterpart node belongs, it also requires additional communication overhead. Moreover, since the distribution of a pair-wise key is made through clusterheads, clusterheads can impersonate communicating nodes.

\section{Pair-Wise Key Agreement Scheme Based on the Cluster Structure and Secret Sharing}

To begin with, it is assumed that each node exchanges its own public key and clusterhead information with other nodes, by attaching them to the routing message. In addition, each node attaches a clue $\left(g^{f\left(i d_{i}\right)}\right)$ of its secret share into the routing message to prove its ownership. The security issues and solutions for routing messages have been addressed in a lot of literatures including [3]. However, these issues are beyond the scope of this paper.

The proposed scheme is based on the following assumptions.

- Dealer generates a polynomial $f(x)=a_{k-1} x^{k-1}+a_{k-2} x^{k-2}+\ldots+a_{0}(\bmod p)$.

- Each node knows a generator $\mathrm{g}$ of $\mathrm{Z}_{p}^{*}$.

- Each node receives $g^{a_{0}}, g^{a_{1}}, \ldots, g^{a_{k-1}}$ that are witness of the coefficients of the sharing polynomial from the dealer.

- Each node receives its secret share $\left(\mathrm{S}_{\mathrm{i}}=f\left(\mathrm{id}_{\mathrm{i}}\right)(\bmod \mathrm{p})\right)$ through an out-of-band method before entering Ad-hoc network. Each node employs its secret share to prove the ownership of its public key.

- Each node generates its public/private key pair and stores them after entering Adhoc network.

The proposed scheme for a pair-wise key agreement is as follows. For the sake of convenience, let's assume that node A wants to make a key agreement with node B belonging to other cluster.

1 Node A generates its random number $\left(R_{A} \in Z_{p}^{*}\right)$ and computes a Diffie-Hellman value $\left(g^{R_{A}}\right)$ for the key agreement. It encrypts only the Diffie-Hellman value, the source, and destination field in the key agreement packet with the public key of node $\mathrm{B}$ and adds an encapsulation header to the encrypted packet. The source of the encapsulation header is the clusterhead of node $\mathrm{A}$ and the destination of the encapsulation header is the clusterhead of node B. Node A sends the capsulated packet to its clusterhead.

2 The clusterhead of node A forwards the capsulated packet to the clusterhead of node $\mathrm{B}$ according to the routing path.

3 The intermediate nodes between the clusterheads of node A and B forward this capsulated packet along the established routing path. 
4 The clusterhead of node B strips off the encapsulation header and broadcasts the encrypted inner packet.

5 Each member node decrypts the encrypted packet with its private key. Since the packet was encrypted with the public key of node B, only node B can decrypt the packet and achieve the Diffie-Hellman value of node $\mathrm{A}\left(g^{R_{A}}\right)$.

6 Node B repeats from step 1 to step 4 after setting node B to the source and node A to the destination.

\section{Security and Performance Analyses}

Our scheme is based on the Diffie-Hellman key exchange protocol. The DiffieHellman protocol is inherently vulnerable to a man-in-the-middle attack. However, since the proposed scheme forces a node to encrypt its Diffie-Hellman value with the counterpart's public key, it is very difficult for intermediate nodes to accomplish manin-the-middle attacks without the corresponding private key. Furthermore, the proposed scheme hides the entities of key agreement by encrypting the source and destination field of the key agreement packet. Only the clusterheads of the source and destination of the key agreement are known through the encapsulation header. As a result, an adversary should know all private keys within the destination's cluster to launch a man-in-the-middle attack successfully.

Let's assume that $n$ is the number of nodes, there are $c$ compromised nodes holding $k$ private keys of other nodes, and $l$ is the number of cluster members belonging to a destination's cluster. If $c$ compromised nodes exchange their key sets with one another, the probability $p_{l}$ that all private keys of cluster members belonging to the destination's cluster are exposed to the $c$ nodes is as follow.

$$
p_{l}=\left(1-\left(\frac{{ }_{n-1-l} C_{k}}{{ }_{n-1} C_{k}}\right)^{c}\right)^{l}
$$

To evaluate the efficiency of the proposed scheme, an event-driven simulator for Ad-hoc networks was developed. The simulation has been done with 25 and 45 nodes on a plane $500 \mathrm{~m} \times 500 \mathrm{~m}$ during 600 seconds. Initially, 25 nodes were assumed to spread randomly on the plane, and they were assumed to move to any direction at any time randomly. The period of key agreement was set to 3 seconds and the entities of key agreement were selected randomly. Next, our simulation has been extended with 45 nodes while other parameters have been the same.

The proposed scheme was compared with the CABM[2]. The average number of sent messages was measured as the radio transmission range increased. The simulation result in Figure 1 shows that the proposed scheme reduces the number of sent messages significantly as compared with the CABM. It is also shown that the proposed scheme doesn't increase the number of sent messages so much though the number of nodes increases. 


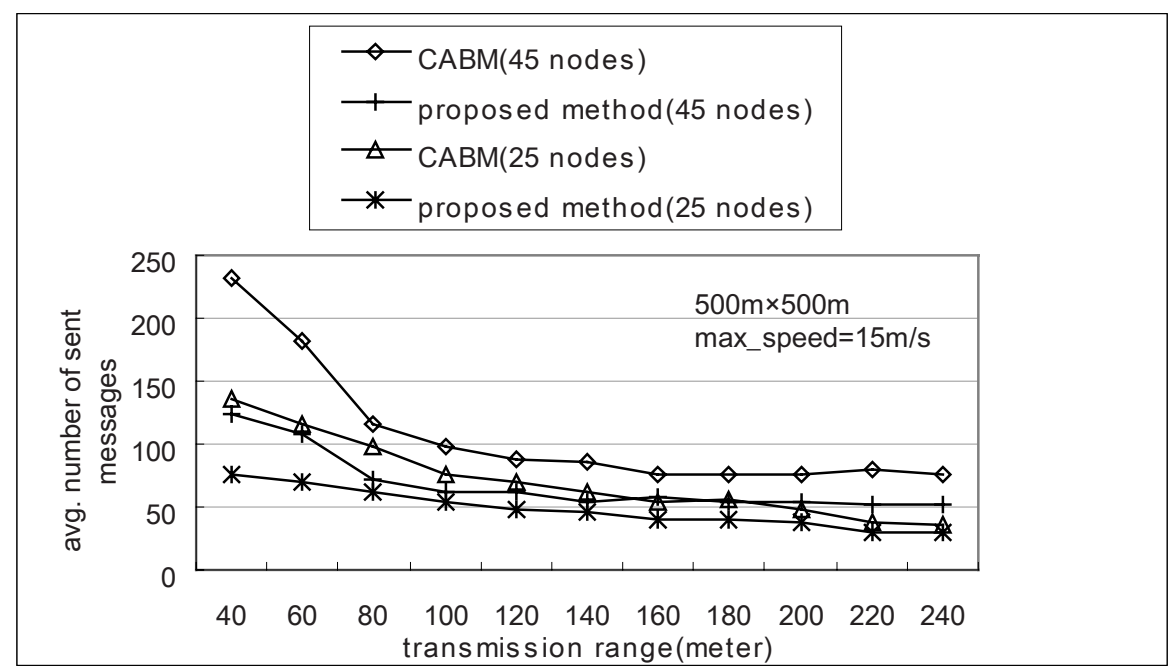

Fig. 1. Average number of sent messages against radio transmission range

\section{Conclusion}

In this paper, a pair-wise key agreement scheme based on the cluster structure and the verifiable secret sharing scheme has been proposed. Since the scheme forces each source to encrypt the source, the destination, and the Diffie-Hellman value in its key agreement packet with the destination's public key, it is very difficult for intermediate nodes to accomplish man-in-the-middle attacks. Also, the proposed scheme hides the entities of a key agreement using encapsulation header to prevent an adversary holding a few private keys from launching a man-in-the-middle attack easily. The simulation result shows that the proposed scheme offers a significant improvement over the CABM.

\section{References}

1. Schoenmakers, B.: A Simple Publicly Verifiable Secret Sharing Scheme and its Application to Electronic Voting. CRYPTO '99. Lecture Notes in Computer Science. Vol. 1666. Springer-Verlag, Berlin Heidelberg New York (1999) 148-164

2. Venkatraman, L., Agrawa, D.P.: A Novel Authentication scheme for Ad-hoc Networks. Proc. of IEEE WCNC 2000. Vol. 3. (2000) 1268-1273

3. Sanzgiri, K., Dahill, B., Levine, B., Shields, C., Belding-Royer, E.: A Secure Routing Protocol for Ad-hoc Networks. Proc. of ICNP'02. (2002) 78-87 